\title{
Bazo errante gigante con torsión del pedículo e infarto esplénico: Reporte de un caso
}

\author{
Ronald Yesid Maestre Serrano ${ }^{1}$, Hanner Acevedo-Reyes ${ }^{1}$, \\ Silvio Cohen-Ríos ${ }^{1}$ y Jarib Álvarez-Jiménez ${ }^{1,2}$
}

\section{Giant Wandering Spleen with torsion of the pedicle and splenic infarction: A case report}

Background: The wandering spleen is an uncommon clinical entity. Its clinical spectrum varies from asymptomatic disease to associated complications and its management is predominantly by surgery. Clinical case: Man with clinical picture of pain and palpable abdominal mass, with tomographic findings suggestive of pathology with retroperitoneal seating, with secondary intestinal obstruction; by laparotomy incidentally, an ectopic spleen is identified, only fixed through a torsioned vascular pedicle and signs of portal hypertension, performing splenectomy. Conclusions: Splenic torsion is the most frequent complication of the errant spleen, a very rare entity with very few cases published in Colombia.

Key words: ectopic spleen; splenectomy; splenomegaly; spleen; acute abdomen.

\section{Resumen}

Introducción: El bazo errante, es una entidad clínica poco común. Su espectro clínico varía desde enfermedad asintomática hasta complicaciones asociadas y su manejo es predominantemente quirúrgico. Caso clínico: Hombre, con cuadro clínico de dolor y masa abdominal palpable, con hallazgos tomográficos sugestivos de patología con asiento en retroperitoneo, con obstrucción intestinal secundaria; por laparotomía se identifica de forma incidental bazo ectópico solo fijado a través de pedículo vascular torsionado y signos de hipertensión portal, realizándose esplenectomía. Conclusiones: La torsión esplénica es la complicación más frecuente del bazo errante, una entidad bastante rara con muy pocos casos publicados en Colombia. Palabras clave: bazo ectópico; esplenectomía; esplenomegalia; bazo; abdomen agudo.

\section{Introducción}

El bazo errante o bazo ectópico, tiene muy baja incidencia; se caracteriza por hipermovilidad del bazo debido a la laxitud o ausencia de los ligamentos normales que unen el bazo al cuadrante superior izquierdo. En consecuencia, el bazo baja al abdomen inferior por la fuerza de la gravedad, unida solo a su pedículo vascular anormalmente alargado. La presentación clínica puede variar desde un hallazgo incidental hasta un abdomen agudo. Dentro de las complicaciones en la literatura se enumera la torsión de su pedículo, la compresión de otro órgano por el bazo o el pedículo y la susceptibilidad del bazo al trauma. Los síntomas en esta patología se presentan cuando hay complicaciones relacionadas con la torsión irreductible que produce congestión venosa, infarto arterial, estrangulación y ruptura del bazo. La primera opción de tratamiento es la esplenopexia para un bazo errante no complicado o complicado, pero viable y esplenectomía para un bazo no viable. Es más frecuente en la niñez especialmente antes del primer año, con un segundo pico de incidencia en la tercera década de la vida, afectando más a mujeres que a hombres ${ }^{1,2}$. A continuación, se presenta el caso de un paciente con torsión esplénica con bazo errante en mesogastrio gigante y síntomas de dolor recurrente y oclusión mecánica al tracto digestivo, quien recibió abordaje multidisciplinario y manejo quirúrgico.
'Universidad Libre Seccional Barranquilla, Facultad de Ciencias de la Salud. Barranquilla, Colombia. ${ }^{2}$ Clínica General del Norte. Barranquilla, Colombia.

Recibido el 31 de julio de 2018 y aceptado para publicación el 27 de agosto de 2018.

Correspondencia a:

Dr. Ronald Yesid Maestre Serrano

rmaestre@unilibrebaq.edu.co 

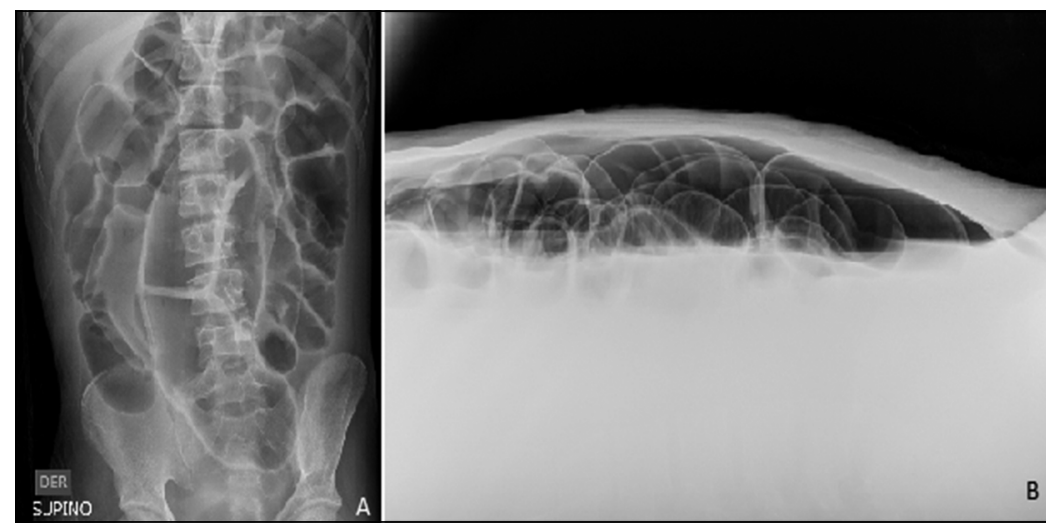

Figura 1. Hallazgos radiológicos de la serie de abdomen inicial. A: Proyección en decúbito supino con rayo anteroposterior que revela distensión del colon y de asas intestinales delgadas por íleo, gas en región rectal, edema interasas. B: Niveles hidroaéreos en posición en decúbito supino con rayo horizontal.

Figura 2. Corte axial de tomografía computada en el que se evidencia como la masa ocupa todo el diámetro transverso de la cavidad abdominal.
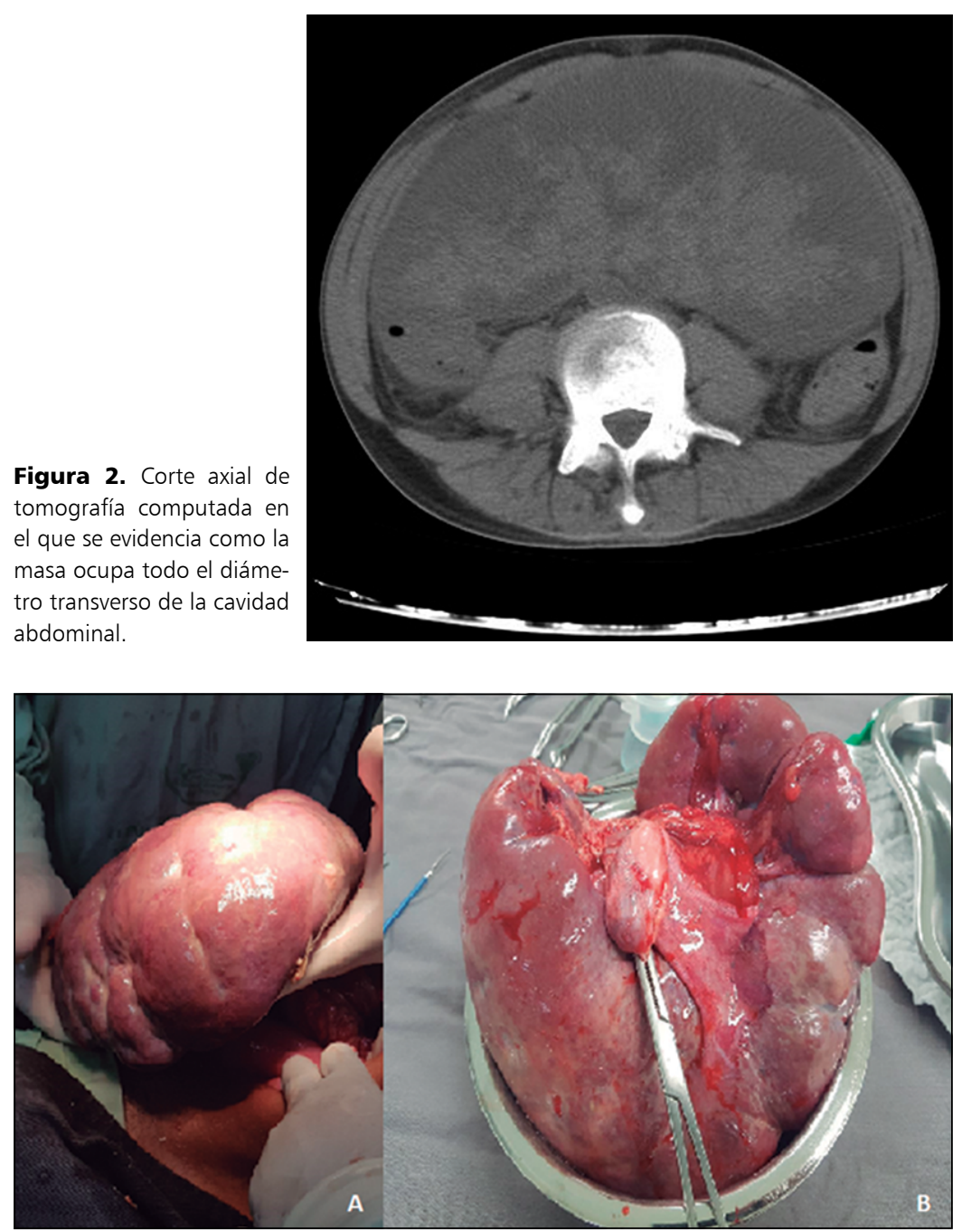

Figura 3. A: Superficie externa de bazo ectópico aumentado de tamaño. B: Superficie interna de espécimen con pedículo vascular complaciente.

\section{Presentación del caso}

Paciente masculino de 17 años de edad, con retardo mental por antecedente perinatal de microcefalia y secuelas neurológicas asociadas a dependencia y postración en cama. Por déficit neurológico, el interrogatorio fue respondido por los padres, quienes manifestaron que el paciente presentaba dolor abdominal diez días previos a la consulta, además de disminución en la frecuencia de deposiciones y deseo de ingesta de alimentos. El paciente no tenía indicación quirúrgica urgente y durante el examen físico se le palpó masa abdominal gigante, reportada por estudio tomográfico extrainstitucional como una masa peritoneal localizada en hemiabdomen derecho y extendida hasta fosa iliaca izquierda; adicionalmente, el paciente se encontraba en malas condiciones generales y musculonutricionales, con agregados pulmonares, sin signos de irritación peritoneal que motivaron la decisión de intervención quirúrgica urgente, por lo que fue hospitalizado con restricción de vía oral, aporte de líquidos, electrolitos y calorías basales vía endovenosa. Se solicitaron estudios paraclínicos que arrojaron los siguientes resultados: hemograma con hemoglobina $8,1 \mathrm{~g} / \mathrm{dl}$; hematocrito $25,0 \%$; leucocitos 33.850 células $/ \mathrm{mm}^{3}$; plaquetas 613.000 células $/ \mathrm{mm}^{3}$; tiempos de coagulación TP 16,1 segundos; INR 1,5; TPT 39,1 segundos; creatinina 1,47 mg/dl; BUN $25,5 \mathrm{mg} / \mathrm{dl}$; cloro $122 \mathrm{mEq} / \mathrm{L}$; sodio $154 \mathrm{mEq} / \mathrm{L}$; potasio $3,4 \mathrm{mEq} / \mathrm{L}$; glucosa $99 \mathrm{mg} / \mathrm{dl}$.

Los estudios imagenológicos de Rayos X seriado de abdomen inicial, arrojaron como resultado una importante dilatación de asas de intestino delgado, lo que es un hallazgo consistente con obstrucción intestinal (Figura 1).

Se realizó corrección endovenosa de hipokalemia y administración parenteral de vitamina $\mathrm{k}$ para normalización de tiempos de coagulación que se encontraban ligeramente prolongados. Así mismo, $\mathrm{TC}$ de abdomen que reportó masa retroperitoneal de origen inespecífico (Figura 2), por lo que se decidió agendar para biopsia de lesión por vía laparoscópica.

Durante estancia en quirófano, se decidió realizar resección quirúrgica de masa con abordaje vía laparotomía mediana supra e infraumbilical, con hallazgos quirúrgicos de bazo ectópico de localización mesogástrica flotante por ausencia de fijaciones peritoneales con rotación y alargamiento de su pedículo vascular gigante con dimensiones no especificadas en la descripción operatoria, pero evidente en estudios de imágenes y con signos de hipertensión portal, realizándose esplenectomía más lavado peritoneal, sin complicaciones (Figura 3 ).

El paciente presentó evolución tórpida de su 
posoperatorio inmediato y mediato, con descenso de las cifras de hemoglobina a $7 \mathrm{~g} / \mathrm{dl}$, requiriendo transfusión de hemoderivados y con ausencia de flatos y deposiciones, además, de distensión abdominal, recibiendo inicialmente manejo expectante, pero decidiéndose reintervención quirúrgica al quinto día de su postoperatorio, por evidencia radiológica de niveles hidroaéreos, relaparotomizando y evidenciando adherencias de omento a ángulo esplénico de colon, realizándose lisis de adherencias peritoneales y cierre de pared abdominal, con evolución tórpida inicial, pero posterior mejoría al manejo médico expectante; período en el que fue valorado por especialidades clínicas, quienes realizaron diagnóstico de trombosis esplénica e indicaron tratamiento con evolución satisfactoria hasta el egreso.

\section{Discusión}

El bazo errante (BE) es una entidad poco común que tiene su origen en una alteración congénita o adquirida en el desarrollo del mesogastrio dorsal que produce ausencia, desarrollo anormal o hiperlaxitud del soporte ligamentoso que une el bazo al cuadrante superior izquierdo: ligamento gastroesplénico, ligamento esplenorrenal y ligamento frenocólico. Esto permite que el bazo descienda al abdomen inferior por la fuerza de gravedad, adoptando una posición anormal dentro de la cavidad abdominal ${ }^{1,2}$. Esta laxitud ligamentosa favorece la presencia de una mayor movilidad esplénica que permite que el bazo se sitúe libremente en la cavidad abdominal, situación que predispone a complicaciones ${ }^{3,4}$.

En Colombia, solo existe un reporte previo de caso de bazo errante ${ }^{3} y$ existen muy pocos reportes de casos a nivel de Latinoamérica, lo que subraya la importancia del presente caso clínico en nuestro entorno.

La primera descripción detallada de esta entidad clínica fue hecha por Van Horne en 1667, como hallazgo incidental en una autopsia. No se conoce con exactitud la incidencia real de esta afección a nivel mundial; sin embargo, existen reportes de incidencia por este evento de $0,16 \%$; aunque en series de autopsias descritas su frecuencia aumenta hasta el 10-30\%. El bazo errante suele presentarse con mayor frecuencia en niños menores de 1 año y en adultos entre los 20 y 40 años, especialmente en mujeres con respecto a hombres en una proporción de $20: 1^{1,5}$. La sintomatología generalmente es vaga e inespecífica, aunque en caso de torsión de su pedículo vascular puede llegar a producir un cuadro de abdomen agudo, lo cual se considera una eventua- lidad poco común ${ }^{5}$. Otros factores que incrementan la movilidad del bazo son: laxitud de la pared abdominal, distensión gástrica, efectos hormonales del embarazo, multiparidad y esplenomegalia ${ }^{6}$.

En la actualidad, el tratamiento de elección consiste en la destorsión y en la esplenopexia, siempre y cuando no exista compromiso irreversible de la irrigación esplénica, en cuyo caso debe realizarse esplenectomía independientemente de la edad del paciente. La esplenopexia se puede realizar mediante diferentes técnicas: fijando la cápsula esplénica al cuadrante superior izquierdo o a la pared abdominal anterior; usando o no malla de ácido poliglicólico. En los últimos años se ha descrito la destorsión y la esplenopexia con malla mediante laparoscopia con resultados aparentemente similares a la cirugía abierta $^{4}$.

El retraso en el diagnóstico puede producir, además de absceso esplénico, hemorragia por varices gastroesofágicas y necrosis de la cola del páncreas. Se ha descrito la relación con vólvulo de páncreas y gástrico, quiste dermoide, eventración diafragmática derecha y oclusión del eje celíaco, lo cual indica una etiología concomitante.

En la actualidad, dado que la mortalidad intraoperatoria es inferior al $1 \%$ y a que se han descrito complicaciones hasta del $65 \%$ de los casos no tratados quirúrgicamente, la tendencia es cada vez más a la esplenectomía, considerándose en la actualidad una técnica segura ${ }^{7}$. Se han publicado un reducido número de casos de abordaje laparoscópico de una torsión esplénica, utilizándose especialmente en casos de urgencia diferida o de cirugía programada.

Las condiciones asociadas con el bazo errante incluyen agrandamiento o ausencia de un riñón, esplenomegalia y embarazo previo. Se cree que la esplenomegalia y el embarazo contribuyen a la laxitud de la estructura de soporte por efecto directo de la gravedad y los estrógenos, respectivamente ${ }^{1}$.

\section{Conclusión}

La torsión esplénica es la complicación más frecuente del bazo errante, una entidad bastante rara con muy pocos casos publicados en Colombia, que suele diagnosticarse ante la aparición de complicaciones asociadas, o de manera incidental. En la mayoría de los casos se asocia a otras patologías abdominales, pero no se describe asociación con alteraciones neurológicas. Nuestro paciente recibió manejo quirúrgico vía laparotomía, por la imprecisión diagnóstica inicial y por el tamaño del bazo que impedía adecuada fijación parietal. 


\section{CASOS CLÍNICOS}

\section{Responsabilidades éticas}

Protección de personas y animales. Los autores declaran que para esta investigación no se han realizado experimentos en seres humanos ni en animales.

Confidencialidad de los datos. Los autores declaran que en este artículo no aparecen datos de pacientes.
Conflicto de intereses: Los autores declaran no tener ningún conflicto de interés.

Agradecimientos: Expresamos agradecimientos a la Clínica General del Norte en la ciudad de Barranquilla (Colombia).

Financiación: No fuentes de financiación.

\section{Referencias}

1. Blouhos K, Boulas KA, Salpigktidis I, Barettas N. Ectopic spleen: An easily identifiable but commonly undiagnosed entity until manifestation of complications. International Journal of Surgery Case Reports 2014;5:451-4. https://doi.org/10.1016/j.ijscr.2014.05.010.

2. Viana C, Cristino H, Veiga C, Leão P. Splenic torsion, a challenging diagnosis: case report and review of literature. International Journal of Surgery Case Reports 2018;44:212-6 https://doi org/10.1016/j.ijscr.2018.02.032.
3. Aleán-Argueta LF, Londoño-Arévalo MA, Morales-Cárdenas AM, Puentes-Velosa S, Uriza-Carrasco LF, Rubio-Bermeo OD. Torsión e infarto esplénico: reporte de caso. Universitas Medica 2013;54: 361-8.

4. Pérez-Legaz J, Moya-Forcén P, Oller I, Arroyo A, Calpena R. Bazo ectópico. Cirugía urgente o electiva? Cirugía Española 2013;91:344-5.

5. Planellas Giné P, Farrés Coll R, Rodríguez-Hermosa JI, Pujadas de Palol M, Codina-Cazador A. Abordaje laparoscópico de abdomen agudo por torsión esplénica. Laparoscopy of acute abdomen due to splenic torsion. Cirugía Española 2012;90:61-3. https://doi. org/10.1016/j.ciresp.2010.12.016.

6. Prieto-Montaño JJ, Salazar W, Reyna-Villasmil E, Santos-Bolívar J. Torsión de bazo ectópico. Torsion of a wandering spleen. Cirugía Española 2009;86:324-5. https://doi.org/10.1016/j. ciresp.2009.01.025.

7. Ramos-Clemente JI, Pérez-Ramos MA, Benavides-Fernández A, MaraverGarcía A. Torsión esplénica en paciente con bazo ectópico. Cirugía Española 2012;90:126-7. https://doi.org/10.1016/j. ciresp.2010.10.015. 